\title{
HOLONOMY RIGIDITY FOR RICCI-FLAT METRICS
}

\author{
BERND AMMANN, KLAUS KRÖNCKE, HARTMUT WEISS, AND FREDERIK WITT
}

\begin{abstract}
On a closed connected oriented manifold $M$ we study the space $\mathcal{M}_{\|}(M)$ of all Riemannian metrics which admit a non-zero parallel spinor on the universal covering. Such metrics are Ricci-flat, and all known Ricci-flat metrics are of this form. We show the following: The space $\mathcal{M}_{\|}(M)$ is a smooth submanifold of the space of all metrics, and its premoduli space is a smooth finite-dimensional manifold. The holonomy group is locally constant on $\mathcal{M}_{\|}(M)$. If $M$ is spin, then the dimension of the space of parallel spinors is a locally constant function on $\mathcal{M}_{\|}(M)$.
\end{abstract}

\section{Overview OVER THE RESUlts}

Let $M$ be a compact connected oriented manifold without boundary, and let $\pi$ : $\widetilde{M} \rightarrow M$ be its universal covering. We assume throughout the article that $\widetilde{M}$ is spin. We define $\mathcal{M}_{\|}(M)$ to be the space of all Riemannian metrics on $M$, such that $(\widetilde{M}, \tilde{g}), \tilde{g}:=\pi^{*} g$, carries a (non-zero) parallel spinor. This implies that $g$ is a stable Ricci-flat metric on $M$, see [6]. It is still an open question, whether $\mathcal{M}_{\|}(M)$ contains all Ricci-flat metrics on $M$.

In the past the space of such metrics was studied in much detail in the irreducible and simply-connected case, see e.g. [17, [18. Much less is known already in the non-simply-connected irreducible case, see e.g. 19. However, the general reducible case has virtually not been addressed at all. For example there is no classification of the full holonomy groups for metrics in $\mathcal{M}_{\|}(M)$ if the restricted holonomy is reducible.

The second named author recently found an efficient method to describe deformations of products of stable Ricci-flat manifolds [12]. In the present article we are using this method to see that the moduli space $\mathcal{M}_{\|}(M)$ is well-behaved.

In fact we show that $\mathcal{M}_{\|}(M)$ is a smooth submanifold of the space of all metrics, and its premoduli space is a smooth finite-dimensional manifold, see Corollary 4. If we view the dimension of the space of parallel spinors as a function $\mathcal{M}_{\|}(M) \rightarrow \mathbb{N}_{0}$, then this function is locally constant, see Corollary 3 . Similarly, the full holonomy group, viewed as a function from $\mathcal{M}_{\|}(M)$ to conjugacy classes of subgroups in $\mathrm{GL}(n, \mathbb{R})$ is locally constant, see Theorem 1 .

\section{RiCCI-FLAT METRICS}

Conventions. All our manifolds will be connected and of dimension $n \geq 3$ unless stated otherwise. A spin manifold is a manifold together with a fixed spin structure.

A Riemannian manifold $(M, g)$ is Ricci-flat if its Ricci tensor Ric ${ }^{g}$ vanishes identically. There are various reasons to study this class of metrics.

It is already a challenging problem to decide whether a compact manifold admits a Ricci-flat metric. Compact manifolds with positive Ricci curvature must have finite fundamental group due to the Bonnet-Myers theorem. A similar obstruction is also available for compact manifolds with nonegative Ricci curvature: as a consequence

Date: September 19, 2018. 
of the splitting theorem by Cheeger and Gromoll [5], one obtains the following theorem, see also Fischer and Wolf [7].

Theorem (Structure theorem for Ricci-flat manifolds). Let $(M, g)$ be a compact Ricci-flat manifold. Then there exists a finite normal Riemannian covering $\bar{M} \times$ $T^{q} \rightarrow M$ with $(\bar{M}, \bar{g})$ a compact simply-connected Ricci-flat manifold and $\left(T^{q}, g_{f l}\right)$ a flat torus.

The theorem implies that $\pi_{1}(M)$ contains a free abelian group $\mathbb{Z}^{q}$ of rank $q$ of finite index, and $q$ satisfies $b_{1}(M) \leq q \leq \operatorname{dim} M$, which acts by translations on $\mathbb{R}^{q}$ and trivially on $\bar{M}$.

A second reason for studying Ricci-flat metrics comes from the intimate relation with the concept of holonomy. To fix notation we briefly recall the definition of holonomy groups and some of their main properties. We refer to [3, Chapter 10] for further details. Let us fix a point $x \in M$ and an identification of $T_{x} M$ with $\mathbb{R}^{n}$. Up to conjugacy in $\operatorname{GL}(n, \mathbb{R})$ these choices define a $\operatorname{subgroup} \operatorname{Hol}(M, g) \subset \operatorname{GL}(n, \mathbb{R})$ as the set of endomorphisms given by parallel transport around a loop in $x$, called the (full) holonomy group of $(M, g)$. The subgroup obtained by taking loops homotopic to the constant path is called the restricted holonomy group and is denoted by $\operatorname{Hol}_{0}(M, g)$. Obviously, $\operatorname{Hol}_{0}(M, g)$ is a normal and connected subgroup of $\operatorname{Hol}(M, g)$, and we have $\operatorname{Hol}(\widetilde{M}, \tilde{g})=\operatorname{Hol}_{0}(M, g)$. It is also known that $\operatorname{Hol}_{0}(M, g)$ is a closed Lie subgroup of $\mathrm{SO}(n)$, in contrast to $\operatorname{Hol}(M, g)$ which might be non-compact, see [20]. Parallel transport induces a group epimorphism $\pi_{1}(M) \rightarrow \operatorname{Hol}(M, g) / \operatorname{Hol}_{0}(M, g)$, and thus $\operatorname{Hol}(M, g) / \operatorname{Hol}_{0}(M, g)$ is countable. As a consequence $\operatorname{Hol}_{0}(M, g)$ is the connected component of $\operatorname{Hol}(M, g)$ containing the identity, and the index of $\operatorname{Hol}_{0}(M, g)$ in $\operatorname{Hol}(M, g)$ is the number of connected components of $\operatorname{Hol}(M, g)$.

For a locally irreducible, non-symmetric and Ricci-flat Riemannian manifold $(M, g)$ of dimension $n, \operatorname{Hol}_{0}(M, g)$ is conjugate to one of the groups occuring in Table 1 The possible full holonomy groups of such manifolds were classified by McInnes 14, see Wang 19 for the spin case. In the general Ricci-flat case, however, it is difficult to determine the full holonomy group. The full holonomy group of a non-irreducible Ricci-flat spin manifold does not have to be a product of holonomy groups in McInnes' list. Still, it follows from the structure theorem for Ricci-flat manifolds, that the subgroup $\mathbb{Z}^{q} \subset \pi_{1}(M)$ is in the kernel of the map $\pi_{1}(M) \rightarrow$ $\operatorname{Hol}(M, g) / \operatorname{Hol}_{0}(M, g)$. Thus $\operatorname{Hol}(M, g) / \operatorname{Hol}_{0}(M, g)$ is finite, see also [5, Theorem $6]$.

A third reason comes from the connection with spin geometry (see for instance 9 for more background on spinors). A parallel spinor on any Riemannian spin manifold forces the underlying metric to be Ricci-flat with holonomy group strictly contained in $\mathrm{SO}(n)$. Conversely, as we have just recalled, a locally irreducible orientable Ricciflat manifold is either of holonomy $\mathrm{SO}(n)$, or it has a simply-connected restricted holonomy group. In the latter case case the universal covering of $M$ is spin and there is a non-trivial parallel spinor on the universal covering. The dimension of the space of parallel spinors $\mathcal{P}(M, g)$ is determined by the holonomy group [17, see Table 1. Furthermore, if the compact manifold $(M, g)$ carries a parallel spinor, then it follows from Wang's work [18] that one obtains a parallel spinor for any metric in the connected component of $g$ within the space of Ricci-flat metrics on $M$.

In the whole article a spinor is a smooth section of the complex spinor bundle and $\operatorname{dim} \mathcal{P}(M, g)$ is the complex dimension of the space of parallel spinors.

Our main theorem studies the holonomy group of a metric with parallel spinor under Ricci-flat deformations. 
Theorem 1 (Rigidity of the holonomy group). Let $(M, g)$ be a compact Riemannian manifold whose universal covering is spin and carries a parallel spinor. If $g_{t}$, $t \in I:=[0, T]$ is a smooth family of Ricci-flat metrics such that $g_{0}=g$, then $\operatorname{Hol}\left(M, g_{t}\right)$ is conjugate to $\operatorname{Hol}(M, g)$ in $\operatorname{GL}(n, \mathbb{R})$.

\section{Rigidity OF PRODUCTS}

An important ingredient in the proof of Theorem 1 is the following product formula by Kröncke 12. It essentially tells us that product metrics $g \times h$ on $N \times P$, with $g \in \mathcal{M}_{\|}(N), h \in \mathcal{M}_{\|}(P)$ and $N, P$ compact, are rigid in the sense that deformations within $\mathcal{M}_{\|}(N \times P)$ are again product metrics. This cannot hold in the strict sense, as flat tori and pull-backs of $g \times h$ by non-product diffeomorphisms provide counterexamples to rigidity in the strict sense. However, rigidity holds modulo diffeomorphisms, provided that one of the factors does not carry parallel vector fields.

To describe this in detail, let $g$ be a Riemannian metric on a compact manifold $N$. An infinitesimal deformation of $g$ given by a symmetric 2-tensor $h$ is orthogonal to the conformal class if $h$ is trace-free (i.e. $\operatorname{tr}_{g} h=0$ ), and $h$ is orthogonal to the diffeomorphism orbit of $g$ if $h$ is divergence-free. On trace-free, divergence-free symmetric 2-tensors the linearization of the Ricci curvature functional $g \mapsto \operatorname{Ric}^{g}$ is given by the Einstein operator $\Delta_{E}^{N}=\frac{1}{2} \nabla^{*} \nabla-\stackrel{\circ}{R}$, where $\stackrel{\circ}{R} h(X, Y):=\sum h\left(R_{e_{i}, X} Y, e_{i}\right)$ for a frame $\left(e_{i}\right)$ for $g$. For any smooth family $g_{t}$ of Ricci-flat metrics of some fixed volume such that $g_{0}=g$ and such that $h=\left.\frac{d}{d t}\right|_{t=0} g_{t}$ is divergence-free, $h$ is in addition trace-free and $\Delta_{E}^{N} h=0$, see [3, Chapter 12].

If a trace-free, divergence-free symmetric 2 -tensor $h$ is in $\operatorname{ker}\left(\Delta_{E}^{N}\right)$, then it is called an infinitesimal Ricci-flat deformation. An infinitesmal Ricci-flat deformation $h$ is called integrable if there exists a smooth family $g_{t}$ of Ricci-flat metrics such that $g_{0}=g$ and $\left.\frac{d}{d t}\right|_{t=0} g_{t}=h$. We say that $g$ is a stable Ricci-flat metric if $\Delta_{E}^{N}$ is positive-semidefinit on trace-free, divergence-free symmetric 2-tensors. All metrics in $\mathcal{M}_{\|}(N)$ are stable, see [18] and also [6].

Theorem (Ricci-flat deformations of products, [12, Prop. 4.5 and 4.6]). If $\left(N^{n}, g\right)$ and $\left(P^{p}, h\right)$ are two stable Ricci-flat manifolds, then $(N \times P, g+h)$ is also stable. Furthermore, on trace-free, divergence-free symmetric 2-tensors we have

$$
\operatorname{ker}\left(\Delta_{E}^{N \times P}\right)=\mathbb{R}(p \cdot g-n \cdot h) \oplus\left(\Gamma_{\|}(T N) \odot \Gamma_{\|}(T P)\right) \oplus \operatorname{ker}\left(\Delta_{E}^{N}\right) \oplus \operatorname{ker}\left(\Delta_{E}^{P}\right),
$$

where $\Gamma_{\|}$is the space of parallel sections. Thus, if all infinitesimal Ricci-flat deformations of $(N, g)$ and $(P, h)$ are integrable, then all infinitesimal Ricci-flat deformations of $(N \times P, g+h)$ are integrable.

In particular, if $\Gamma_{\|}(T N)=0$ or if $\Gamma_{\|}(T P)=0$, then Ricci-flat deformations of $g \times h$ are up to diffeomorphisms again of product form.

\begin{tabular}{|l|c|}
\hline $\operatorname{Hol}\left(M^{n}, g\right)$ & $\operatorname{dim} \mathcal{P}(M, g)$ \\
\hline $\mathrm{SO}(n)$ & - \\
$\mathrm{SU}(m), n=2 m$ & 2 \\
$\mathrm{Sp}(k), n=4 k$ & $k+1$ \\
$\mathrm{Spin}(7), n=8$ & 1 \\
$\mathrm{G}_{2}, n=7$ & 1 \\
\hline
\end{tabular}

TABLE 1. Special holonomy groups and the dimension of the space of parallel spinors. 


\section{Rigidity OF THE RESTRICTED HOLONOMY GROUP}

In order to prove the theorem we first prove the analogous statement for the restricted holonomy.

Proposition 2 (Rigidity of the restricted holonomy group). Let $(M, g)$ be a compact Riemannian manifold whose universal covering is spin and carries a parallel spinor. If $g_{t}, t \in I:=[0, T]$ is a smooth family of Ricci-flat metrics such that $g_{0}=g$, then $\operatorname{Hol}_{0}\left(M, g_{t}\right)$ is conjugate to $\operatorname{Hol}_{0}(M, g)$ in $\mathrm{GL}(n, \mathbb{R})$, i.e. there are $Q_{t} \in \operatorname{GL}(n, \mathbb{R})$ with $\operatorname{Hol}_{0}\left(M, g_{t}\right)=Q_{t} \operatorname{Hol}_{0}(M, g) Q_{t}^{-1}$. Moreover, the map $t \mapsto Q_{t}$ can be chosen continuously.

In the proposition we have fixed a base point $x \in M$ and an identification $T_{x} M \cong \mathbb{R}^{n}$. Thus $\operatorname{Hol}_{0}\left(M, g_{t}\right) \in \mathrm{GL}(n, \mathbb{R})$.

\section{Remark.}

(i) The proposition (and our main theorem) apply for example to Riemannian spin manifolds carrying a parallel spinor. Under these assumptions, the universal covering $(\widetilde{M}, \tilde{g})$ is spin as well, and the parallel spinor on $(M, g)$ lifts to a parallel spinor on $(\widetilde{M}, \tilde{g})$.

(ii) The proposition (and our main theorem) are false for non-compact manifolds. Indeed, let $g$ be a (possibly non-complete) Ricci-flat metric on $\mathbb{R}^{n}$ with a parallel spinor and non-trivial holonomy group $H$. Let $\mu_{s}: \mathbb{R}^{n} \rightarrow \mathbb{R}^{n}$ be multiplication with $1-s$ for $1>s \geq 0$. Then $g_{s}:=(1-s)^{-2} \mu_{s}^{*} g$ also has holonomy $H$ for $1>s \geq 0$. However, for $s \rightarrow 1$ the metric $g_{s}$ converges in the compact-open $C^{\infty}$ topology to the flat metric $g_{1}$ with trivial holonomy.

Proof of the proposition. Consider the universal Riemannian covering $(\widetilde{M}, \tilde{g})$ of $(M, g)$, and let $\Gamma \cong \pi_{1}(M)$ be the group of Deck transformations. Using the structure theorem for Ricci-flat metrics [5, 7] recalled in Section 2, we know that $(M, g)$ has a finite normal Riemannian covering isometric to $(\bar{M}, \bar{g}) \times\left(T^{q}, g_{f l}\right)$, where $(\bar{M}, \bar{g})$ is compact, simply-connected and Ricci-flat, and $\left(T^{q}, g_{f l}\right)$ a flat torus. By the de Rham decomposition theorem, $(\bar{M}, \bar{g})$ is globally isometric to a Riemannian product of compact, simply-connected and irreducible Ricci-flat manifolds $\left(\bar{M}_{i}, \bar{g}_{i}\right)$, $i=1, \ldots, r$. Thus we obtain a finite Riemannian covering

$$
\left(\bar{M} \times T^{q}=\bar{M}_{1} \times \ldots \times \bar{M}_{r} \times T^{q}, \bar{g}_{1} \times \ldots \times \bar{g}_{r} \times g_{f l}\right) \rightarrow(M, g)
$$

whose holonomy group equals $H_{1} \times \ldots \times H_{r}=\operatorname{Hol}(\widetilde{M}, \tilde{g})=\operatorname{Hol}_{0}(M, g)$.

Since $\widetilde{M}$ is spin, so is $\bar{M}$, and thus all $\bar{M}_{i}$ are spin. The spin structure on each $\bar{M}_{i}$ is unique as $\bar{M}_{i}$ is simply-connected. On $T^{q}$ there are several distinct spin structures; we choose the unique spin structure that admits parallel spinors. A parallel spinor on a product corresponds to a product of parallel spinors (this follows from simple representation theoretic considerations as in [1, Proposition 4.5], see for instance 13. Theorem 2.5] for a detailed proof). Thus the given parallel spinor on $\widetilde{M}$ implies the existence of parallel spinors on each $\bar{M}_{i}$. The holonomy group $H_{i}:=\operatorname{Hol}\left(\bar{M}_{i}, \bar{g}_{i}\right)$ is therefore conjugate to one of the groups of Table 1.

Then by taking products, we obtain a parallel spinor on $\left(\bar{M} \times T^{q}, \bar{g} \times g_{f l}\right)$, which lifts to a parallel spinor on $\widetilde{M}$, and we can assume without loss of generality that this is the given parallel spinor on $\widetilde{M}$ discussed above.

Next consider the family of Ricci-flat metrics $g_{t}$ on $M$. The covering $\bar{M} \times T^{q} \rightarrow M$ can be chosen independently of $t$. And let $\bar{g}_{t} \times g_{f l, t}$ be the pullback of $g_{t}$ to $\bar{M} \times T^{q}$ induced by $\left(M, g_{t}\right)$. The metric $\bar{g}=\bar{g}_{1} \times \ldots \times \bar{g}_{r}$ is a product of irreducible Ricci-flat metrics which admit a parallel spinor, and thus the metrics $\bar{g}_{i}$ are stable. The factors $\left(\bar{M}_{i}, \bar{g}_{i}\right)$ are irreducible, hence defined by a torsion-free $G$-structure for one 
of the groups $G \neq \mathrm{SO}(n)$ taken from Table 1 It then follows from 16 (building on work of [10]) that infinitesimal Ricci-flat deformations of $\bar{g}_{i}$ are integrable. Finally, they admit no harmonic 1-forms as simply-connectedness implies vanishing of the first Betti number $b_{1}$. Therefore the theorem on Ricci-flat deformations of products in Section 3 implies that the Ricci-flat deformation $\bar{g}_{t}$ of $\bar{g}$ is - up to pull-back by diffeomorphisms in $\operatorname{Diff}_{0}(\bar{M})$ - of the form $\bar{g}_{t, 1} \times \ldots \times \bar{g}_{t, r}$, where $\bar{g}_{t, i}$ is a smooth Ricci-flat deformation of $\bar{g}_{i}$. By the known rigidity results in the irreducible case [16, 18, the holonomy group of nearby Ricci-flat deformations of $\bar{g}_{i}$ will be conjugate to $H_{i}$, and the conjugating element can be chosen continuously in $t$.

\section{Applications}

Corollary 3. Let $M$ be a compact spin manifold, and let $g_{0} \in \mathcal{M}_{\|}(M)$. If $g_{t}$, $t \in I:=[0, T]$ is a smooth family of Ricci-flat metrics on $M$ such that $g_{0}=g$, then $g_{t} \in \mathcal{M}_{\|}(M)$ and $\operatorname{dim} \mathcal{P}\left(M, g_{t}\right)$ is constant in $t$.

Remark. A parallel spinor is a special case of a real Killing spinors. If $(M, g)$ carries a (non-zero) real Killing spinor, then $g$ is Einstein. However, it was shown in [4, that our results do no longer hold if we replace parallel spinors by real Killing spinors and Ricci-flat metrics by Einstein metrics.

Proof. In the proof of Proposition 2 we have chosen a spin structure on $\bar{M} \times T^{q}$. As $\left(M, g_{t}\right)$ carries a parallel spinor, this spin structure has to coincide with the pullback of the spin structure on $M$.

Let $\bar{\Gamma}$ be the (finite) group of Deck transformations of the covering space $\bar{M} \times T^{q} \rightarrow$ $M$. By normality of this covering space, $\bar{\Gamma}$ acts transitively on each fiber, and this action lifts to the spin structure, and thus to the spinor bundle. The dimension of $\mathcal{P}\left(\bar{M} \times T^{q}, \bar{g}_{t} \times g_{f l, t}\right)$ is determined by the holonomy group $\operatorname{Hol}\left(\bar{M}, \bar{g}_{t}\right)=\operatorname{Hol}(\bar{M} \times$ $\left.T^{q}, \bar{g}_{t} \times g_{f l}\right)=\operatorname{Hol}_{0}\left(M, g_{t}\right)$ which therefore does not depend on $t$. Further, $\mathcal{P}\left(M, g_{t}\right)$ can be identified with the subspace of $\mathcal{P}\left(\bar{M} \times T^{q}, \bar{g}_{t} \times g_{f l, t}\right)$ which is invariant for the representation $\rho_{t}: \bar{\Gamma} \rightarrow \operatorname{GL}\left(\mathcal{P}\left(\bar{M} \times T^{q}, \bar{g}_{t} \times g_{f l, t}\right)\right)$. Representation theory of finite groups implies

$$
\operatorname{dim} \mathcal{P}\left(M, g_{t}\right)=\frac{1}{|\bar{\Gamma}|} \sum_{\gamma \in \bar{\Gamma}} \operatorname{Tr} \rho_{t}(\gamma)
$$

which is continuous in $t$, hence locally constant.

This corollary extends Wang's result from [18] which states that the dimension is constant if $M$ is simply-connected and $g_{0}$ irreducible. An immediate consequence is that the premoduli space $\mathcal{M}_{\|}(M) / \operatorname{Diff}_{0}(M)$ is an open subset of the premoduli space of Ricci-flat metrics. In general, the premoduli space of Ricci-flat metrics is merely a real analytic subset of a finite-dimensional real analytic manifold, and the tangent space of this manifold can be identified with the space of infinitesimal Ricci-flat deformations and rescalings of the metric (cf. for instance the discussion in [3. Chapter 12.F] building on Koiso's work [11]).

Corollary 4. Assume that $M$ is a compact spin manifold. Then $\mathcal{M}_{\|}(M) / \operatorname{Diff}_{0}(M)$ is a smooth manifold. Furthermore, $\mathcal{M}_{\|}(M)$ is a smooth submanifold in the space of all metrics on $M$.

Proof. We have to check that every infinitesimal Ricci-flat deformation of $g_{0} \epsilon$ $\mathcal{M}_{\|}(M)$ is in fact integrable. Consider again the finite cover $\bar{M} \times T^{q} \rightarrow M$. The space of Ricci-flat metrics on $\bar{M} \times T^{q}$ is smooth by the proof of Proposition 2, and so is the space of Ricci-flat metrics on $M$ as the fixed point set of the finite group $\bar{\Gamma}$ acting on the former space by pull-back. This implies the result. 
This generalises Nordström's result [16 for metrics defined by a torsion-free $G$ structure for one of the groups $G \neq \mathrm{SO}(n)$ taken from Table 1 to general metrics in $\mathcal{M}_{\|}(M)$. Nordström makes strong use of Goto's earlier result [10] about the unobstructedness of the deformation theory of such $G$-structures.

A further application involves the spinorial energy functional introduced in 2, to which we refer for details. To define it, consider the space of sections $\mathcal{N}$ of the universal bundle of unit spinors over $M$. A section $\Phi \in \mathcal{N}$ can be thought of as a pair $(g, \phi)$ where $g$ is a Riemannian metric and $\phi \in \Gamma\left(\Sigma_{g} M\right)$ is a $g$-spinor of constant length one. The relevant functional is defined by

$$
\mathcal{E}: \mathcal{N} \rightarrow \mathbb{R}_{\geq 0}, \quad \Phi \mapsto \frac{1}{2} \int_{M}\left|\nabla^{g} \phi\right|_{g}^{2} d v^{g},
$$

where $\nabla^{g}$ denotes the Levi-Civita connection on the $g$-spinor bundle $\Sigma_{g} M,|\cdot|_{g}$ the pointwise norm on $T^{*} M \otimes \Sigma_{g} M$ and $d v^{g}$ the Riemann-Lebesgue measure given by the volume form of $g$. By [2, Corollary 4.10] the set of critical points $\operatorname{Crit}(\mathcal{E})$ consists precisely of $g$-parallel unit spinors $(g, \phi)$, provided that $\operatorname{dim} M>2$. Since by Corollary 3 the dimension of the space of parallel spinors is constant under Ricci-flat deformations, the proof of [2, Theorem 4.17] immediately implies the subsequent

Corollary 5. The functional $\mathcal{E}$ is Morse-Bott, i.e. the critical set $\operatorname{Crit}(\mathcal{E})$ is smooth and $\mathcal{E}$ is non-degenerate transverse to $\operatorname{Crit}(\mathcal{E})$.

Now the universal covering group of $\operatorname{Diff}_{0}(M)$, the diffeomorphisms homotopic to the identity, naturally acts on $\operatorname{Crit}(\mathcal{E})$. It also follows with the arguments above that the premoduli space of critical points, that is, $\operatorname{Crit}(\mathcal{E})$ divided by this action, is smooth as well.

\section{Rigidity OF THE FULL HOLONOMY GROUP}

We now prove Theorem 1, using the following fact from Lie group theory.

Theorem (Montgomery-Zippin [15]). Let $H_{0}$ be a compact subgroup of a Lie group $G$. Then there exists an open neighbourhood $U$ of $H_{0}$ such that if $H$ is a compact subgroup of $G$ contained in $U$, then there exists $g \in G$ with $g^{-1} H g \subset H_{0}$. Moreover, upon sufficiently shrinking $U, g$ can be chosen in any neighbourhood of the identity of $G$.

Proof of Theorem 1, The group epimorphisms

$$
\alpha_{t}: \Gamma=\pi_{1}(M) \rightarrow \operatorname{Hol}\left(M, g_{t}\right) / \operatorname{Hol}_{0}\left(M, g_{t}\right)
$$

factor through $\Gamma \rightarrow \bar{\Gamma}=\Gamma / \mathbb{Z}^{q}$ to epimorphisms $\bar{\alpha}_{t}: \bar{\Gamma} \rightarrow \operatorname{Hol}\left(M, g_{t}\right) / \operatorname{Hol}_{0}\left(M, g_{t}\right)$. Choose loops $\gamma_{1}, \ldots, \gamma_{r}:[0,1] \rightarrow M$ where $\gamma_{i}(0)=\gamma_{i}(1)$ is the base point of $M$ and such that $\bar{\Gamma}=\left\{\left[\gamma_{1}\right], \ldots,\left[\gamma_{\ell}\right]\right\}, \ell=\# \bar{\Gamma}$. Let $A_{t, i}$ be the parallel transport along $\gamma_{i}$ for the metric $g_{t}$.

Thus

$$
\operatorname{Hol}\left(M, g_{t}\right)=A_{t, 1} \cdot \operatorname{Hol}_{0}\left(M, g_{t}\right) \cup \ldots \cup A_{t, \ell} \cdot \operatorname{Hol}_{0}\left(M, g_{t}\right)
$$

Let $k(t):=\#\left\{i \in\{1,2, \ldots, \ell\} \mid A_{t, i} \in \operatorname{Hol}_{0}\left(M, g_{t}\right)\right\}=\# \operatorname{ker} \bar{\alpha}_{t}$. Then the index of $\operatorname{Hol}_{0}\left(M, g_{t}\right)$ in $\operatorname{Hol}\left(M, g_{t}\right)$ is $\ell / k(t)$ and this index is the number of connected components of $\operatorname{Hol}\left(M, g_{t}\right)$. Thus the index of $\operatorname{Hol}_{0}\left(M, g_{t}\right)$ in $\operatorname{Hol}\left(M, g_{t}\right)$ is lower semi-continuous. We fix $t_{0} \in[0, T]$. As $A_{t, i}$ depends continuously on $t$, the theorem of Montgomery-Zippin implies that $\operatorname{Hol}\left(M, g_{t}\right)$ is conjugate to a subgroup $H_{t}$ of $\operatorname{Hol}\left(M, g_{t_{0}}\right)$ if $t$ is sufficiently close to $t_{0}$. Conjugation preserves the connected component containing the identity, thus $\operatorname{Hol}_{0}\left(M, g_{t_{0}}\right) \subset H_{t} \subset \operatorname{Hol}\left(M, g_{t_{0}}\right)$. Lower semi-continuity of the number of connected components of $H_{t}$ implies the result. 


\section{REFERENCES}

[1] F. Adams, Lectures on exceptional Lie groups, ed. by Z. Mahmud and M. Mimura. Chicago Lectures in Mathematics. University of Chicago Press, 1996.

[2] B. Ammann, H. Weiss, And F. Witt, A spinorial energy functional: critical points and gradient flow, to appear in Math. Ann., doi:10.1007/s00208-015-1315-8

[3] A. Besse, Einstein manifolds, Springer, Berlin, 1987.

[4] C. VAn Coevering Deformations of Killing spinors on Sasakian and 3-Sasakian manifolds, arxiv 1301.3479.

[5] J. Cheeger and D. Gromoll, The splitting theorem for manifolds of nonnegative Ricci curvature, J. Differential Geometry 6 (1971/72), 119-128.

[6] X. Dai, X. WAng And G. WeI, On the stability of Riemannian manifold with parallel spinors, Invent. Math. 161 (2005), no. 1, 151-176.

[7] A. Fischer AND J. Wolf, The structure of compact Ricci-flat Riemannian manifolds, J. Diff. Geom. 10 (1975), 277-288.

[8] T. Friedrich, Der erste Eigenwert des Dirac-Operators einer kompakten, Riemannschen Mannigfaltigkeit nichtnegativer Skalarkrümmung, Math. Nachr. 97 (1980), 117-146.

[9] T. FrIEDrich, Dirac operators in Riemannian geometry, Graduate Studies in Mathematics 25, AMS, Providence, 2000.

[10] R. Gото, Moduli spaces of topological calibrations, Calabi-Yau, hyper-Kähler, $G_{2}$ and Spin(7) structures, Internat. J. Math. 15 (2004), 211-257.

[11] N. KoIso, Einstein metrics and complex structures, Invent. Math. 73 (1983), no. 1, 71-106.

[12] K. KRöncke On infinitesimal Einstein deformations, Differ. Geom. Appl. 38 (2015), 41-57.

[13] T. LEISTNER, Lorentzian manifolds with special holonomy and parallel spinors, Preprint No. 486/SFB 288 available at http://www-sfb288.math.tu-berlin.de/Publications/preprint-list/451/500 see also Rend. Circ. Mat. Palermo (2) Suppl. 2002, no. 69, 131-159.

[14] B. MCInNEs, Methods of holonomy theory for Ricci-flat Riemannian manifolds, J. Math. Phys. 32, (1991), 888-896.

[15] D. Montgomery and L. Zippin, A theorem on Lie groups, Bull. Amer. Math. Soc. 48, (1942), 448-452.

[16] J. Nordström, Ricci-flat deformations of metrics with exceptional holonomy, Bull. Lond. Math. Soc. 45 (2013), no. 5, 1004-1018.

[17] M. WAng, Parallel spinors and parallel forms, Ann. Global Anal. Geom. 7 (1989), no. 1, 59-68.

[18] M. WANG, Preserving parallel spinors under metric deformations, Indiana Univ. Math. J. 40 (1991), no. 3, 815-844.

[19] M. WANG, On non-simply connected manifolds with non-trivial parallel spinors, Ann. of Global Anal. Geom. 13 (1995), no. 1, 31-42.

[20] B. Wilking, On compact Riemannian manifolds with noncompact holonomy groups, J. Diff. Geom. 52 (1999), no. 2, 223-257.

Fakultät Für Mathematik, Universität Regensburg, Universitätsstrasse 40, D-93040 Regensburg, Germany

E-mail address: bernd.ammann@mathematik.uni-regensburg.de

Fachbereich Mathematik der Universität Hamburg, Bundesstr. 55, D-20146 Hamburg, GERMANY

E-mail address: klaus.kroencke@uni-hamburg.de

Mathematisches Seminar der Universität Kiel, Ludewig-Meyn Strasse 4, D-24098 Kiel, GERMANY

E-mail address: weiss@math.uni-kiel.de

Institut für Geometrie und Topologie der Universität Stuttgart, Pfaffenwaldring 57, D-70569 Stuttgart, Germany

E-mail address: frederik.witt@mathematik.uni-stuttgart.de 